\title{
Suggestion of Maintenance Criteria for Electric Railroad Facilities Based on Fuzzy TOPSIS
}

\author{
Sunwoo Hwang ${ }^{1}$, Joouk Kim ${ }^{1}$, Hagseoung Kim ${ }^{1}$, Hyungchul Kim ${ }^{2}$ and Youngmin Kim ${ }^{3, *}$ \\ ${ }^{1}$ Innovative Transportation and Logistics Research Center, Korea Railroad Research Institute, Uiwang, 16105, Korea \\ ${ }^{2}$ Smart Electrical \& Signaling Division, Korea Railroad Research Institute, Uiwang, 16105, Korea \\ ${ }^{3}$ Department of Systems Engineering, Ajou University, Suwon, 16499, Korea \\ "Corresponding Author: Youngmin Kim. Email: pretty0m@ajou.ac.kr \\ Received: 21 June 2021; Accepted: 12 August 2021
}

\begin{abstract}
This paper is on the suggestion of maintenance items for electric railway facility systems. With the recent increase in the use of electric locomotives, the utilization and importance of railroad electrical facility systems are also increasing, but the railroad electrical facility system in Korea is rapidly aging. To solve this problem, various methodologies are applied to ensure operational reliability and stability for railroad electrical facility systems, but there is a lack of detailed evaluation criteria for railroad electrical facility system maintenance. Also, maintenance items must be selected in a scientific and systematic method. Therefore, railroad electrical facility systems are selected for study. Design Structure Matrix (DSM) is utilized to establish considerations tailored to the maintenance characteristics, and the Fuzzy-TOPSIS methodology is utilized for determining the maintenance detail evaluation item baseline weights, a multi-criteria decision-making problem. Studies show that degradation, insulation items have the highest weight of $14.63 \%$, and capacity items have the lowest weight of $5.34 \%$. The results of this may be contributed to the underlying research in carrying out maintenance activities to ensure the reliability and safety of railroad electrical facility systems.
\end{abstract}

Keywords: Electrical facility system; railroad maintenance; fuzzy method

\section{Introduction}

With the recent increase in the use of electric railroads, the utilization and importance of railroad electrical facility systems is also increasing. Railroad electrical systems are responsible for enabling power supply and substitution, signal control and communication, and at the subsystem level include subway power, signal control and communications. Currently, Korea's railroad electrical facility system is rapidly aging, and the Ministry of Land, Infrastructure and Transport announced the first basic plan for maintenance management of railroad facilities (2021-2025) as a countermeasure plan [1]. The performance of railroad facility is rated A, B, C, D and E, and Fig. 1 represents the data. Fig. 1a represents the results of the performance assessment of railroad electrical facility systems as of 2020 . The ratio below class $\mathrm{C}$ shows $76.9 \%$ of subway power

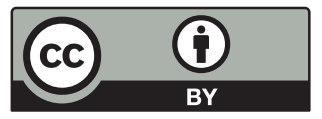

This work is licensed under a Creative Commons Attribution 4.0 International License, which permits unrestricted use, distribution, and reproduction in any medium, provided the original work is properly cited. 
facilities, $51.4 \%$ of signal control facilities, $66.4 \%$ of information and communication facilities, and $11.7 \%$ of subway power facilities, $33.9 \%$ of signal control facilities, and $25 \%$ of information and communication facilities. Fig. $1 \mathrm{~b}$ represents the combined results and forecasts of the performance evaluation of railroad electrical facility systems by 2030 as of 2020 . Overall, the proportion below grade $\mathrm{C}$ is expected to be $83.0 \%$ five years later and $93.4 \% 10$ years later 2030 . In addition, the performance assessment score is expected to be 3.37 in 2025, five years later, and 2.31 in 2030, 10 years later. It can be seen a rapid change from 2025.

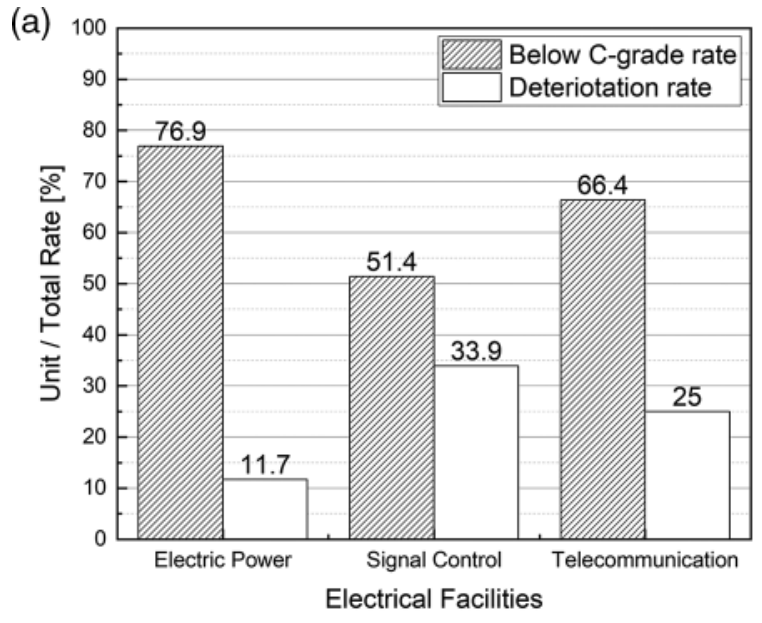

Figure 1: Performance of electrical railroad facility [1] (a) Deteriorated rate of sub system (b) Comprehensive deteriorated rate prediction

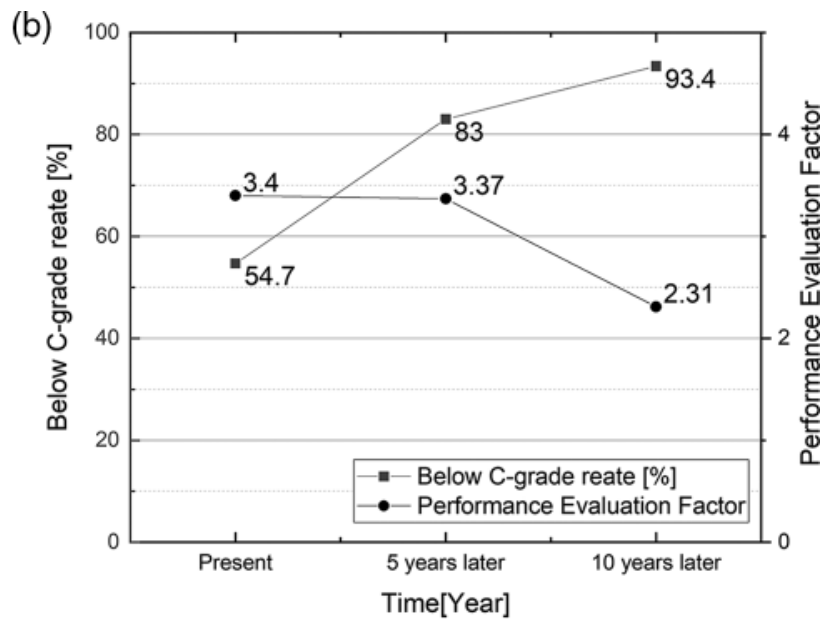

\subsection{Preliminaries}

In line with the need for efficient maintenance of railroad electrical facility systems, underlying studies using various approaches have been conducted. Kang Hyun-il et al. conducted a maintenance optimization survey of railroad operators, conducting a research based on maintenance optimization [2]. Kim Hyun-min and others recognized the problem that $60 \%$ of the maintenance budget is used as labor costs, and suggested the need to improve maintenance through information and automation [3]. They also compared maintenance operations cases in France and the United Kingdom, and conducted studies to improve the efficiency of maintenance operations agencies. Recognizing that maintenance performance does not reflect route-specific weights, Park Hyun-june et al. present the need to derive scientific check cycles through systematic data construction [4]. It was emphasized that data such as obsolescence analysis/recording and follow-up measures should be included to establish a systematic database. In addition, the need for automation of precision diagnosis of facilities was derived by introducing information and communication technologies. With reference to the history of overseas railroad structural reform, Oh Yoon-sic et al. conducted a study on the development of sustainable railroad industry policies from a long-term perspective [5]. In addition, the scale of each railroad investment project, the trend of increase and decrease in maintenance costs, and the ratio of track use to maintenance costs were analyzed. As a result, the overall system, such as national transportation systems, railroad industry structures, safety and environment, should be analyzed rather than simply compared to overseas cases, and efficient maintenance procedures should be prepared. Park Sang-gu and others suggested that the maintenance of railroad facilities remained at a standstill, not meeting the 
trend of railroad advancement after the full opening of the Gyeongbu high speed railroad [6]. To improve this, they present the need to build a database that encompasses information such as railroad facility history management at the national level. The selection of target facilities was made by considering items such as construction cost, train operation impact, other facilities impact, and frequency of failure, and basic items for performance evaluation were prepared. $\mathrm{Na}$ Kyung-min et al. conducted a study on the development of a performance evaluation index analysis program, recognizing that performance evaluation is complex and takes considerable time to calculate the performance evaluation index [7]. The corresponding results lay the foundation for efficiently improving procedures such as complex, time-consuming weight calculations and routespecific parameter calculations in performing subsequent performance evaluations. Recognizing the problem of excessive time spent on data collection and analysis activities in existing performance evaluation procedures, Kang Goune et al. conducted a study on implementing performance evaluation simulations using Virtual Reality (VR) [8]. To ensure the safety of railroad infrastructure, Kim Tae-hyun and others conducted a study on the development of railroad safety information systems and operational architectures through the analysis of technical relevance of usage characteristics and product characteristics reflection/requirements [9]. Choi Kwon-hee and others have conducted quantitative risk assessment studies of railroad safety facilities through analysis of the individual risk (general traveler, passenger crew), Safety Failure Rates (SFF), and diagnostic validity ranges [10]. According to the analysis of the results of the aging and performance evaluation of the railroad electrical facility system proposed earlier, it is time for maintenance of the railroad electrical facility system in Korea. However, maintaining an electrical facility system on all railroad lines currently in operation has limitations in economic and temporal costs, and an assessment item baseline study with systematic and scientific decision-making methods is needed. Furthermore, the maintenance methods currently being performed have an inefficient disadvantage in selecting priorities based on the age of use. Therefore, for the systematic maintenance of railroad electrical facility systems, this paper establishes considerations of the criteria for detailed assessment items for maintenance characteristics and derives weights for maintenance assessment items, Multiple-Criteria Decision-Making (MCDM) problem.

\subsection{Methodology}

Efficient and systematic standards are needed to perform efficient maintenance of aging railroad electrical facility systems, taking into account the limitations of economic and temporal costs, and the establishment of maintenance assessment criteria involves the decision maker's subjective judgment. Because decision-makers' judgments are subjective and ambiguous, a methodology is needed to quantitatively derive opinions from experts in the field. Chen presents a methodology that extends the Technique for Order Performance by Ideal Solution (TOPSIS) logic in a fuzzy environment to solve the MCDM problem [11]. As a result, we have been able to conclude that the application of Fuzzy TOPSIS to the process of solving MCDM problems is effective. we utilize Design Structure Matrix (DSM) and Fuzzy-TOPSIS techniques to determine the weights of the maintenance detail evaluation criteria for railroad electrical facility systems based on the subjective judgment information of decision makers. Fuzzy TOPSIS bases upon the concept that the chosen alternative should have the shortest distance from the positive ideal solution and the farthest from the negative ideal solution. Pushpendra Kumar et al. approached the predict liver disease problem using fuzzy sets and boosting techniques [12], and Yoosef B. Abushark et al. conducted a usability evaluation study on security requirement perspective using the fuzzy AHPTOPSIS approach [13]. Regarding COVID-19, Rupkumar Mahapatra et al. conducted a study to distinguish COVID-19 detected regions by fuzzy directed graphs [14], M. Jayalakshmi et al. 
conducted a health monitoring system study based on fuzzy theory [15]. Yaser Hafeez et al. used fuzzy theory to conduct an approach study for fault detection of distributed components [16]. As such, fuzzy theory has been applied to MCDM problems in many forms in various domains and fields. DSM represents the association of the various components that make up a complex system in matrix, a suitable way to express the interface between each component. we apply the concept of DSM to efficiently solve the MCDM problem in a way that presents the association via qualitative evaluation of the maintenance criteria of railroad electrical facility systems as an interface in addition to the underlying DSM, input-output association method [17]. We find that the Fuji TOPSIS theory is effective in processing ambiguous data. The Fuzzy-TOPSIS technique uses the fuzzy set, fuzzy number's data, and presents the closest alternatives, FPIRP (Fuzzy Positive Ideal Reference Point) and FNIRP (Fuzzy Negative Ideal Reference Point), respectively. First, Fuzzy data is represented by a matrix for alternatives (columns) and criteria (rows) as Eq. (1), the weighted data $W$ of $n$ evaluation criteria by $k$ decision makers are represented as Eq. (2).

$$
\begin{aligned}
& D=\left[\begin{array}{cccc}
x_{11} & x_{12} & \cdots & x_{1 n} \\
x_{21} & x_{22} \cdots & x_{2 n} \\
\vdots & \vdots & \ddots & \vdots \\
x_{m 1} & x_{m 2} & \cdots & x_{m n}
\end{array}\right] \\
& W=\left[w_{1}, w_{2}, \cdots, w_{n}\right]
\end{aligned}
$$

Here, $x$ is the result of evaluating alternative to be chosen by $k$ decision makers as the evaluation criteria. $A$ matrix of combinations $R$ of $x$ and w can be expressed as a normalization matrix $R_{k}$, such as Eqs. (3)-(7), as a membership function for positive fuzzy functions ranging from 0 to 1 , and as $\mu_{A}(x)$, such as Eq. (8).

$$
R_{k}=(a, b, c, d)
$$

$a=\min \left\{a_{k}\right\}$

$b=\frac{1}{K} \sum_{k=1}^{K} b_{k}$

$$
c=\frac{1}{K} \sum_{k=1}^{K} c_{k}
$$

$d=\max \left\{d_{k}\right\}$

$$
\mu_{A}(x)=\left\{\begin{array}{lr}
0, & (x \leq a) \\
\frac{x-a}{b-a}, & (a \leq x \leq b) \\
1, & (b \leq x \leq c) \\
\frac{x-d}{c-d}, & (c \leq x \leq d) \\
0, & (x>d)
\end{array}\right.
$$


We obtain a regularization decision matrix $\mathrm{V}$, weighted by a combination of the fuzzy matrix and the weighted matrix, and distance it from the FPRIP matrix A+ and FNRIP matrix A- for the alternative we want to select preferentially, using the maximum and minimum values for each matrix. The equation for the distance is expressed in Eqs. (9) and (10).

$d^{+}\left(A, A^{+}\right)=\sqrt{1 / K\left[\left(v_{n}^{+}-v_{m n}\right)\right]}$

$\left.d^{-}\left(A, A^{-}\right)=\sqrt{1 / K\left[\left(v_{n}^{-}-v_{m n}\right)\right.}\right]$

Next, we obtain the resulting values for $\mathrm{CC}$, the relative proximity coefficient for each distance, and then, depending on the number of resulting values, we can derive the priorities of the alternatives to be chosen. The equation for obtaining $\mathrm{CC}$ is expressed in Eq. (11). Fig. 2 represents the research flow of this paper.

$C C=d^{-} /\left(d^{+}+d^{-}\right)$

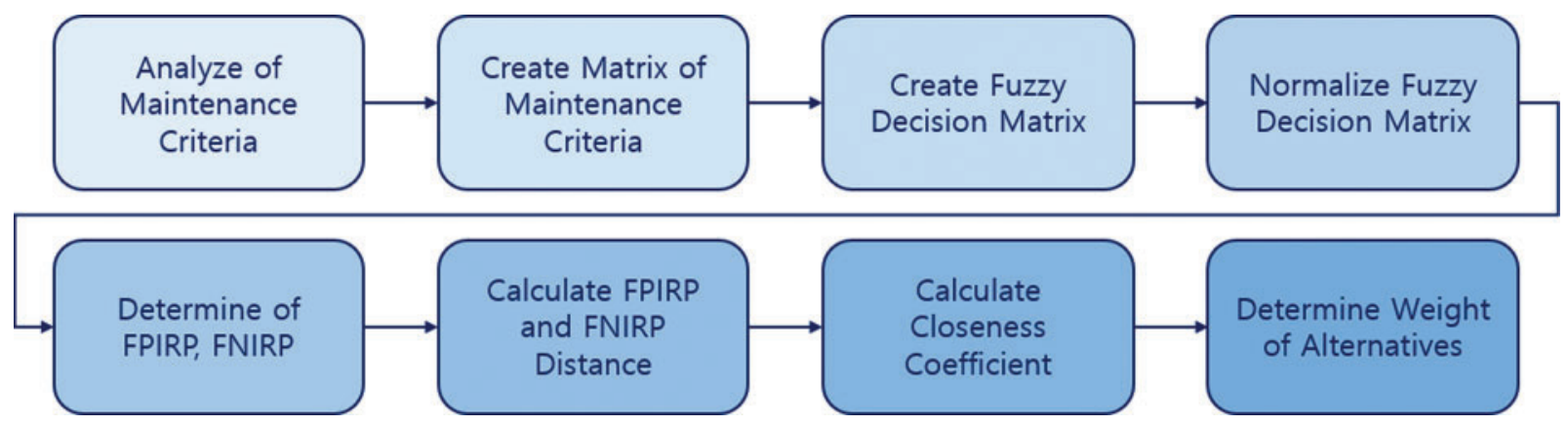

Figure 2: Detailed evaluation item weight determination flow

\section{Maintenance Criteria Analysis}

\subsection{Maintenance Criteria of Electric Railroad Facility System}

Tab. 1 describes safety, durability and usability, which are the criteria for evaluating railroad electrical facility systems. The term safety means "The performance to prevent casualties, damage and loss of railroad facilities under the requirements of railroad facilities," and the term durability means "The performance of railroad facilities to maintain the required functions during the service life of railroad facilities." In addition, usability refers to "The performance of providing adequate convenience and functionality in terms of the use and demand of railroad facilities". Safety, durability, and usability were selected as evaluation criteria, and the detailed criteria for maintenance of railroad electrical facility systems were divided into degradation, insulation, abrasion, strength, noise, corrosion, crack, oil leak, slope, subsidence, elapsed years, number of uses, environment, number of services, number of failure detection, product discontinued, number of maintenance, capacity. 
Table 1: Properties of evaluation criteria

\begin{tabular}{ll}
\hline $\begin{array}{l}\text { Maintenance } \\
\text { criteria }\end{array}$ & Property \\
\hline Safety & $\begin{array}{l}\text { The performance to prevent casualties, damage and loss of rail facilities, } \\
\text { under the requirements of rail facilities }\end{array}$ \\
Durability & $\begin{array}{l}\text { The performance of railroad facilities to maintain the required functions } \\
\text { during the service life of railroad facilities }\end{array}$ \\
Usability & $\begin{array}{l}\text { The performance of providing adequate convenience and functionality in } \\
\text { terms of the use and demand of rail facilities }\end{array}$ \\
\hline
\end{tabular}

To consider the effectiveness of decisions in the selection of maintenance criteria, a process of grouping relatively similar items is needed. Therefore, the association of each criteria was expressed in Design Structure Matrix (DSM) and DSM Partitioning activities were performed to cluster similar items, reflecting the opinions of railroad electrical system maintenance experts. As a result, according to the evaluation criteria safety, durability and usability, there were 10 alternatives, and the results were Figs. 3 and 4.
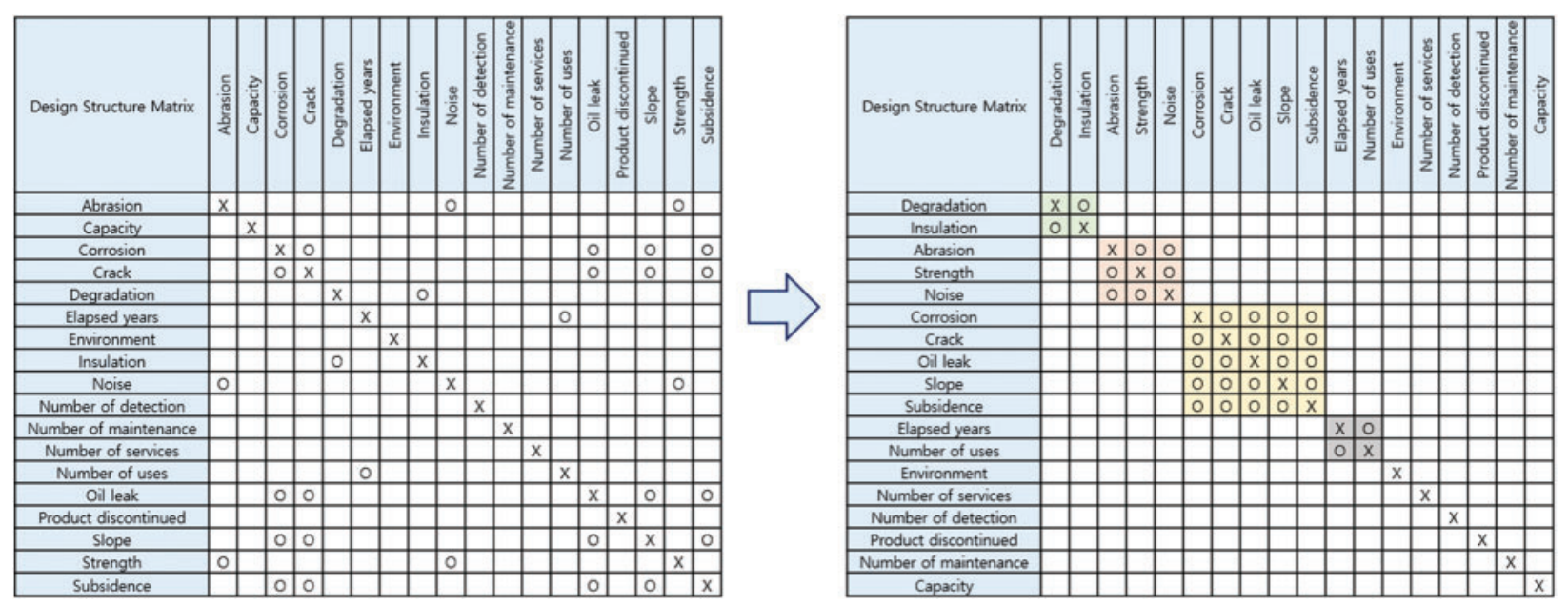

Figure 3: Establishing maintenance criteria using DSM

\subsection{Input Data Setting}

The decision matrices for weight selection of the evaluation criteria were Very Low (VL: 0.0, 0.0, 0.1, 0.2), Low (L: 0.1, 0.2, 0.2, 0.3), Medium Low (ML: 0.2, 0.3, 0.4, 0.5), Medium (M:0.4, 0.5, 0.5, 0.6), Medium High (MH: 0.5, 0.6, 0.7, 0.8), High (H: 0.7, 0.8, 0.8, 0.9), Very High (VH: $0.8,0.9,1.0,1.0)$. The results are expressed in Tab. 2 and Fig. 5.

Decision matrices for alternative weight selection were classified as Very Poor (VP: 0.0, 0.0, 0.1, 0.2), Poor (P: 0.1, 0.2, 0.2, 0.3), Medium Poor (MP: 0.2, 0.3, 0.4, 0.5), Fair (F:0.4, 0.5, 0.5, 0.6), Medium Good (MG: 0.5, 0.6, 0.7, 0.8), Good (G: 0.7, 0.8, 0.8, 0.9), Very Good (VG: 0.8, $0.9,1.0,1.0)$. The results are expressed in Tab. 3 and Fig. 6. 


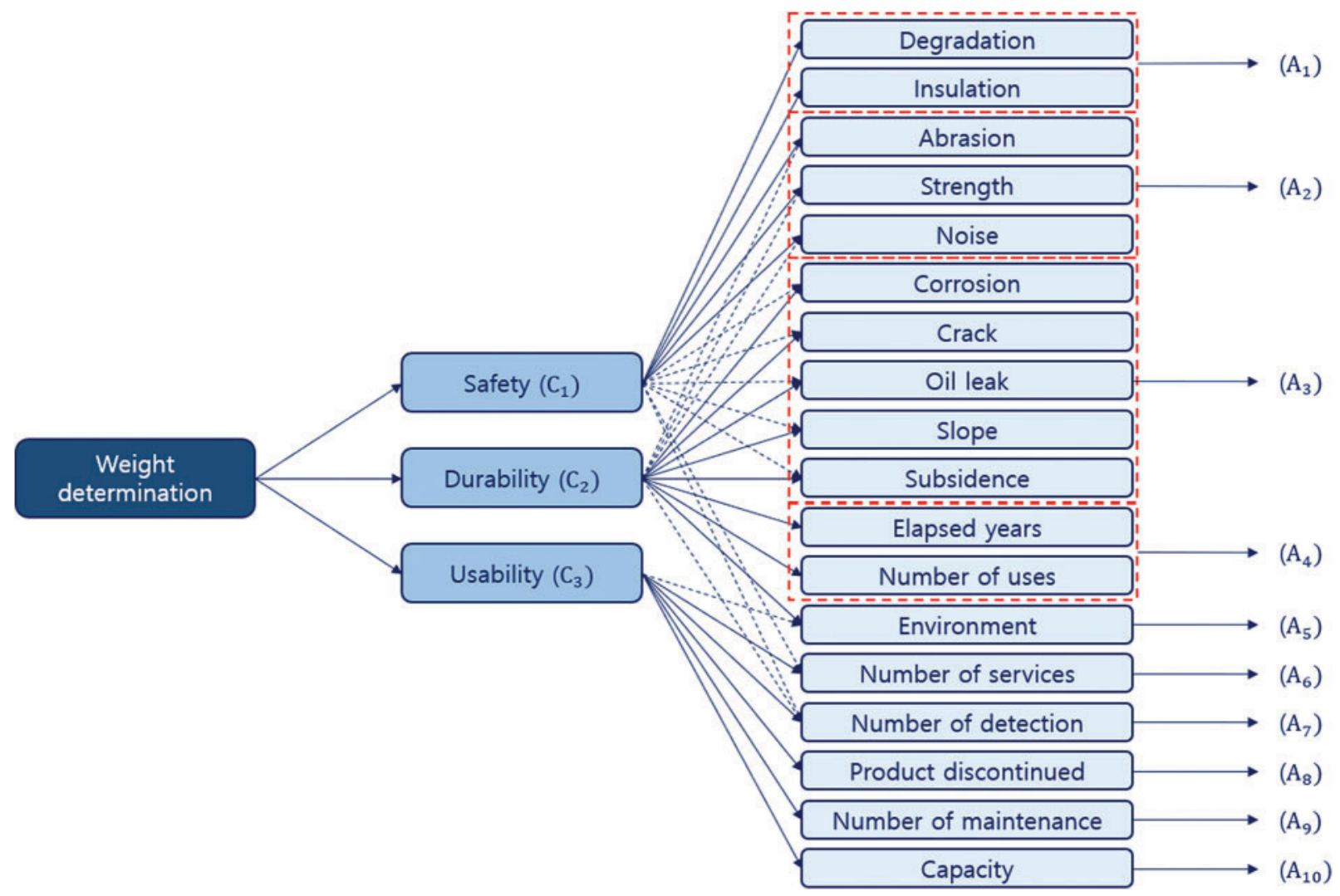

Figure 4: Selection of alternatives to evaluation criteria

Table 2: Linguistic variable for criteria

\begin{tabular}{ll}
\hline Linguistic variable & Fuzzy numbers \\
\hline VL(Very Low) & $(0.0,0.0,0.1,0.2)$ \\
L(Low) & $(0.1,0.2,0.2,0.3)$ \\
ML(Medium Low) & $(0.2,0.3,0.4,0.5)$ \\
M(Medium) & $(0.4,0.5,0.5,0.6)$ \\
MH(Medium High) & $(0.5,0.6,0.7,0.8)$ \\
H(High) & $(0.7,0.8,0.8,0.9)$ \\
VH(Very High) & $(0.8,0.9,1.0,1.0)$ \\
\hline
\end{tabular}

Three railroad electrical system experts were cast to conduct a survey to select the weight of the evaluation criteria for railroad electrical system maintenance and a survey to select the weight of the maintenance criteria, respectively, Tab. 4 and Tabs. 5-7, respectively. Tab. 4 represents the weighting results of decision makers for the evaluation criteria, and Tabs. 5-7 represents the weighting results of decision makers for each alternative for the three evaluation criteria. 


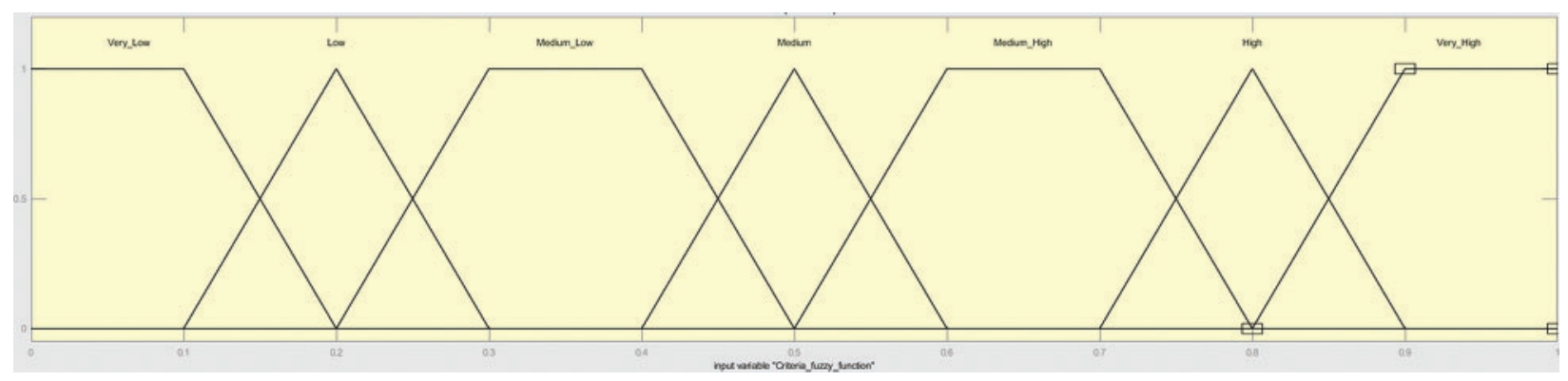

Figure 5: Fuzzy function for evaluation criteria

Table 3: Linguistic variable for alternative

\begin{tabular}{ll}
\hline Linguistic variable & Fuzzy numbers \\
\hline VP(Very Poor) & $(0.0,0.0,0.1,0.2)$ \\
P(Poor) & $(0.1,0.2,0.2,0.3)$ \\
MP(Medium Poor) & $(0.2,0.3,0.4,0.5)$ \\
F(Fair) & $(0.4,0.5,0.5,0.6)$ \\
MG(Medium Good) & $(0.5,0.6,0.7,0.8)$ \\
G(Good) & $(0.7,0.8,0.8,0.9)$ \\
VG(Very Good) & $(0.8,0.9,1.0,1.0)$ \\
\hline
\end{tabular}

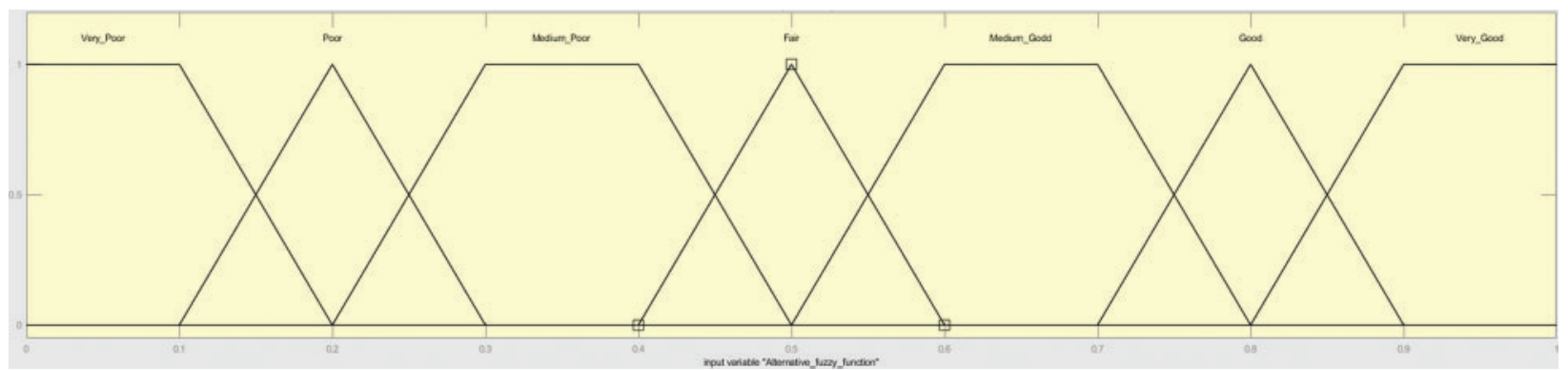

Figure 6: Fuzzy function on alternatives

Table 4: Selection of evaluation criteria weights by decision makers

\begin{tabular}{llll}
\hline Criteria & Expert 1 & Expert 2 & Expert 3 \\
\hline$C_{1}$ & $\mathrm{VH}(0.80,0.90,1.00,1.00)$ & $\mathrm{H}(0.70,0.80,0.80,0.90)$ & $\mathrm{VH}(0.80,0.90,1.00,1.00)$ \\
$C_{2}$ & $\mathrm{H}(0.70,0.80,0.80,0.90)$ & $\mathrm{M}(0.40,0.50,0.50,0.60)$ & $\mathrm{MH}(0.50,0.60,0.70,0.80)$ \\
$C_{3}$ & $\mathrm{MH}(0.50,0.60,0.70,0.80)$ & $\mathrm{M}(0.40,0.50,0.50,0.60)$ & $\mathrm{ML}(0.20,0.30,0.40,0.50)$ \\
\hline
\end{tabular}


Table 5: Selection of alternative weights by decision makers (criteria 1)

\begin{tabular}{lllll}
\hline Criteria & Alternatives & Expert 1 & Expert 2 & Expert 3 \\
\hline$C_{1}$ & $A_{1}$ & $\mathrm{VG}$ & $\mathrm{G}$ & $\mathrm{VG}$ \\
& $A_{2}$ & $\mathrm{MP}$ & $\mathrm{F}$ & $\mathrm{MP}$ \\
& $A_{3}$ & $\mathrm{~F}$ & $\mathrm{G}$ & $\mathrm{F}$ \\
$A_{4}$ & $\mathrm{MG}$ & $\mathrm{MG}$ & $\mathrm{MG}$ \\
& $A_{5}$ & $\mathrm{~F}$ & $\mathrm{P}$ & $\mathrm{MP}$ \\
& $A_{6}$ & $\mathrm{G}$ & $\mathrm{P}$ & $\mathrm{F}$ \\
$A_{7}$ & $\mathrm{MG}$ & $\mathrm{MG}$ & $\mathrm{MP}$ \\
& $A_{8}$ & $\mathrm{G}$ & $\mathrm{G}$ & $\mathrm{F}$ \\
& $A_{9}$ & $\mathrm{MG}$ & $\mathrm{MP}$ & $\mathrm{MG}$ \\
& $A_{10}$ & $\mathrm{P}$ & $\mathrm{P}$ & $\mathrm{P}$ \\
\hline
\end{tabular}

Table 6: Selection of alternative weights by decision makers (criteria 2)

\begin{tabular}{lllll}
\hline Criteria & Alternatives & Expert 1 & Expert 2 & Expert 3 \\
\hline$C_{2}$ & $A_{1}$ & $\mathrm{VG}$ & $\mathrm{VG}$ & $\mathrm{VG}$ \\
& $A_{2}$ & $\mathrm{G}$ & $\mathrm{MP}$ & $\mathrm{P}$ \\
& $A_{3}$ & $\mathrm{~F}$ & $\mathrm{P}$ & $\mathrm{MP}$ \\
$A_{4}$ & $\mathrm{G}$ & $\mathrm{G}$ & $\mathrm{MP}$ \\
& $A_{5}$ & $\mathrm{MP}$ & $\mathrm{G}$ & $\mathrm{MP}$ \\
$A_{6}$ & $\mathrm{G}$ & $\mathrm{P}$ & $\mathrm{F}$ \\
$A_{7}$ & $\mathrm{MG}$ & $\mathrm{G}$ & $\mathrm{MP}$ \\
& $A_{8}$ & $\mathrm{MG}$ & $\mathrm{F}$ & $\mathrm{P}$ \\
& $A_{9}$ & $\mathrm{G}$ & $\mathrm{MP}$ & $\mathrm{MP}$ \\
& $A_{10}$ & $\mathrm{P}$ & $\mathrm{MP}$ & $\mathrm{MP}$ \\
\hline
\end{tabular}

Table 7: Selection of alternative weights by decision makers (criteria 3)

\begin{tabular}{lllll}
\hline Criteria & Alternatives & Expert 1 & Expert 2 & Expert 3 \\
\hline$C_{3}$ & $A_{1}$ & $\mathrm{VG}$ & $\mathrm{G}$ & $\mathrm{MG}$ \\
& $A_{2}$ & $\mathrm{G}$ & $\mathrm{G}$ & $\mathrm{MP}$ \\
& $A_{3}$ & $\mathrm{~F}$ & $\mathrm{P}$ & $\mathrm{F}$ \\
$A_{4}$ & $\mathrm{G}$ & $\mathrm{F}$ & $\mathrm{P}$ \\
& $A_{5}$ & $\mathrm{G}$ & $\mathrm{G}$ & $\mathrm{MP}$ \\
$A_{6}$ & $\mathrm{MG}$ & $\mathrm{F}$ & $\mathrm{F}$ \\
$A_{7}$ & $\mathrm{G}$ & $\mathrm{F}$ & $\mathrm{MG}$ \\
& $A_{8}$ & $\mathrm{G}$ & $\mathrm{MG}$ & $\mathrm{P}$ \\
& $A_{9}$ & $\mathrm{MP}$ & $\mathrm{MG}$ & $\mathrm{P}$ \\
& $A_{10}$ & $\mathrm{MP}$ & $\mathrm{F}$ & $\mathrm{P}$ \\
\hline
\end{tabular}




\section{Calculation of Weights for Maintenance Criteria}

\subsection{Fuzzification of Decision Matrix}

Tab. 8 is a fuzzy matrix quantitatively represented by applying Eqs. (4)-(7) to the results of Tabs. 5-7. Weights for criterion $1(0.70,0.87,0.93,1.00)$, weights for criterion $2(0.40,0.63,0.67$, $0.90)$, and weights for criterion $3(0.20,0.47,0.53,0.80)$. Tab. 9 represents the result of multiplying the weight with the fuzzy data for the alternative in Tab. 8.

Table 8: Quantification results of weights and alternatives to evaluation criteria

\begin{tabular}{llll}
\hline Alternative & Criterion 1 & Criterion 2 & Criterion 3 \\
\hline$A_{1}$ & $(0.70,0.87,0.93,1.00)$ & $(0.80,0.90,1.00,1.00)$ & $(0.50,0.77,0.83,1.00)$ \\
$A_{2}$ & $(0.20,0.37,0.43,0.60)$ & $(0.10,0.43,0.47,0.90)$ & $(0.20,0.63,0.67,0.90)$ \\
$A_{3}$ & $(0.40,0.60,0.60,0.90)$ & $(0.10,0.33,0.37,0.60)$ & $(0.10,0.40,0.40,0.60)$ \\
$A_{4}$ & $(0.50,0.60,0.70,0.80)$ & $(0.20,0.63,0.67,0.90)$ & $(0.10,0.50,0.50,0.90)$ \\
$A_{5}$ & $(0.10,0.33,0.37,0.60)$ & $(0.20,0.47,0.53,0.90)$ & $(0.20,0.63,0.67,0.90)$ \\
$A_{6}$ & $(0.10,0.50,0.50,0.90)$ & $(0.10,0.50,0.50,0.90)$ & $(0.40,0.53,0.57,0.80)$ \\
$A_{7}$ & $(0.20,0.50,0.60,0.80)$ & $(0.20,0.57,0.63,0.90)$ & $(0.40,0.63,0.67,0.90)$ \\
$A_{8}$ & $(0.40,0.70,0.70,0.90)$ & $(0.10,0.43,0.47,0.80)$ & $(0.10,0.53,0.57,0.90)$ \\
$A_{9}$ & $(0.20,0.50,0.60,0.80)$ & $(0.20,0.47,0.53,0.90)$ & $(0.10,0.37,0.43,0.80)$ \\
$A_{10}$ & $(0.10,0.20,0.20,0.30)$ & $(0.10,0.27,0.33,0.50)$ & $(0.10,0.33,0.37,0.60)$ \\
Weight & $(0.70,0.87,0.93,1.00)$ & $(0.40,0.63,0.67,0.90)$ & $(0.20,0.47,0.53,0.80)$ \\
\hline
\end{tabular}

Table 9: Matrix of decision fuzzy data weighted by evaluation criteria

\begin{tabular}{llll}
\hline Alternative & Criterion 1 & Criterion 2 & Criterion 3 \\
\hline$A_{1}$ & $(0.49,0.75,0.87,1.00)$ & $(0.32,0.57,0.67,0.90)$ & $(0.10,0.36,0.44,0.80)$ \\
$A_{2}$ & $(0.14,0.32,0.40,0.60)$ & $(0.04,0.27,0.31,0.81)$ & $(0.04,0.30,0.36,0.72)$ \\
$A_{3}$ & $(0.28,0.52,0.56,0.90)$ & $(0.04,0.21,0.24,0.54)$ & $(0.02,0.19,0.21,0.48)$ \\
$A_{4}$ & $(0.35,0.52,0.65,0.80)$ & $(0.08,0.40,0.44,0.81)$ & $(0.02,0.23,0.27,0.72)$ \\
$A_{5}$ & $(0.07,0.29,0.34,0.60)$ & $(0.08,0.30,0.36,0.81)$ & $(0.04,0.30,0.36,0.72)$ \\
$A_{6}$ & $(0.07,0.43,0.47,0.90)$ & $(0.04,0.32,0.33,0.81)$ & $(0.08,0.25,0.30,0.64)$ \\
$A_{7}$ & $(0.14,0.43,0.56,0.80)$ & $(0.08,0.36,0.42,0.81)$ & $(0.08,0.30,0.36,0.72)$ \\
$A_{8}$ & $(0.28,0.61,0.65,0.90)$ & $(0.04,0.27,0.31,0.72)$ & $(0.02,0.25,0.30,0.72)$ \\
$A_{9}$ & $(0.14,0.43,0.56,0.80)$ & $(0.08,0.30,0.36,0.81)$ & $(0.02,0.17,0.23,0.64)$ \\
$A_{10}$ & $(0.07,0.17,0.19,0.30)$ & $(0.04,0.17,0.22,0.45)$ & $(0.02,0.16,0.20,0.48)$
\end{tabular}

\subsection{FPIRP and FNIRP Determination}

Based on the results for Tab. 9, determine the most ideal answer FPIRP and the least ideal answer FNIRP for each evaluation criteria and all alternatives. This process treats each evaluation criteria as a column, determining the maximum value FPIRP with the Eq. given in Eq. (9), and the minimum value FNIRP with given in Eq. (10). The results for this were shown as Tab. 10. 
Table 10: Determination result of FPIRP and FNIRP

\begin{tabular}{llll}
\hline Criteria & Criterion 1 & Criterion 2 & Criterion 3 \\
\hline$A^{+}$ & $(1.00,1.00,1.00,1.00)$ & $(0.90,0.90,0.90,0.90)$ & $(0.80,0.80,0.80,0.80)$ \\
$A^{-}$ & $(0.07,0.07,0.07,0.07)$ & $(0.04,0.04,0.04,0.04)$ & $(0.02,0.02,0.02,0.02)$ \\
\hline
\end{tabular}

\section{Determination of Weights for Maintenance Criteria}

Find the displacement between FPIRP and FNIRP determined for each evaluation criteria from the fuzzy data presented in Tab. 10. Hereby, the results of the maximum and minimum fuzzy data of the alternatives for each evaluation criteria can be found. The calculations showed that Tab. 11. Based on the results of Tab. 11, the distances of FPIRP and FNIRP for each alternative are calculated to take into account the criteria safety, durability, and usability. Based on the calculated results, we calculate the $\mathrm{CC}$ value using Eq. (11). Normalization can be performed according to the $\mathrm{CC}$ value to determine the percentage value for each alternative. By normalizing the results for this, the weights of alternatives for each railroad electrical facility maintenance detail criteria item are presented as Percentage. As a result, degradation, insulation items were weighted the highest at $14.63 \%$, and capacity items were weighted the lowest at $5.34 \%$. Results for this are shown in Tab. 12 and Fig. 7.

Table 11: Calculate the distance of alternatives to the criteria

\begin{tabular}{llll}
\hline FPIRP & Criterion 1 & Criterion 2 & Criterion 3 \\
\hline $\mathrm{d}\left(A_{1}, A^{+}\right)$ & 0.291 & 0.353 & 0.451 \\
$\mathrm{~d}\left(A_{2}, A^{+}\right)$ & 0.656 & 0.609 & 0.509 \\
$\mathrm{~d}\left(A_{3}, A^{+}\right)$ & 0.488 & 0.666 & 0.598 \\
$\mathrm{~d}\left(A_{4}, A^{+}\right)$ & 0.451 & 0.533 & 0.552 \\
$\mathrm{~d}\left(A_{5}, A^{+}\right)$ & 0.701 & 0.579 & 0.509 \\
$\mathrm{~d}\left(A_{6}, A^{+}\right)$ & 0.608 & 0.594 & 0.523 \\
$\mathrm{~d}\left(A_{7}, A^{+}\right)$ & 0.569 & 0.548 & 0.494 \\
$\mathrm{~d}\left(A_{8}, A^{+}\right)$ & 0.448 & 0.614 & 0.540 \\
$\mathrm{~d}\left(A_{9}, A^{+}\right)$ & 0.569 & 0.579 & 0.582 \\
$\mathrm{~d}\left(A_{10}, A^{+}\right)$ & 0.822 & 0.696 & 0.611 \\
$\mathrm{~d}\left(A_{1}, A^{-}\right)$ & 0.733 & 0.611 & 0.477 \\
$\mathrm{~d}\left(A_{2}, A^{-}\right)$ & 0.339 & 0.425 & 0.412 \\
$\mathrm{~d}\left(A_{3}, A^{-}\right)$ & 0.542 & 0.283 & 0.263 \\
$\mathrm{~d}\left(A_{4}, A^{-}\right)$ & 0.537 & 0.471 & 0.386 \\
$\mathrm{~d}\left(A_{5}, A^{-}\right)$ & 0.317 & 0.436 & 0.412 \\
$\mathrm{~d}\left(A_{6}, A^{-}\right)$ & 0.495 & 0.435 & 0.361 \\
$\mathrm{~d}\left(A_{7}, A^{-}\right)$ & 0.477 & 0.459 & 0.413 \\
$\mathrm{~d}\left(A_{8}, A^{-}\right)$ & 0.583 & 0.384 & 0.394 \\
$\mathrm{~d}\left(A_{9}, A^{-}\right)$ & 0.477 & 0.436 & 0.336 \\
$\mathrm{~d}\left(A_{10}, A^{-}\right)$ & 0.139 & 0.233 & 0.255 \\
\hline
\end{tabular}


Table 12: Determination of weights by calculating closeness coefficient

\begin{tabular}{lllll}
\hline Alternative & $d_{i}^{+}$ & $d_{i}^{-}$ & $\mathrm{CC}$ & Weight [\%] \\
\hline$A_{1}$ & 1.095 & 1.820 & 0.624 & 14.63 \\
$A_{2}$ & 1.774 & 1.175 & 0.399 & 9.34 \\
$A_{3}$ & 1.752 & 1.088 & 0.383 & 8.98 \\
$A_{4}$ & 1.536 & 1.395 & 0.476 & 11.15 \\
$A_{5}$ & 1.789 & 1.165 & 0.394 & 9.25 \\
$A_{6}$ & 1.725 & 1.290 & 0.428 & 10.03 \\
$A_{7}$ & 1.611 & 1.349 & 0.456 & 10.68 \\
$A_{8}$ & 1.603 & 1.362 & 0.459 & 10.77 \\
$A_{9}$ & 1.730 & 1.249 & 0.419 & 9.83 \\
$A_{10}$ & 2.128 & 0.628 & 0.228 & 5.34 \\
\hline
\end{tabular}

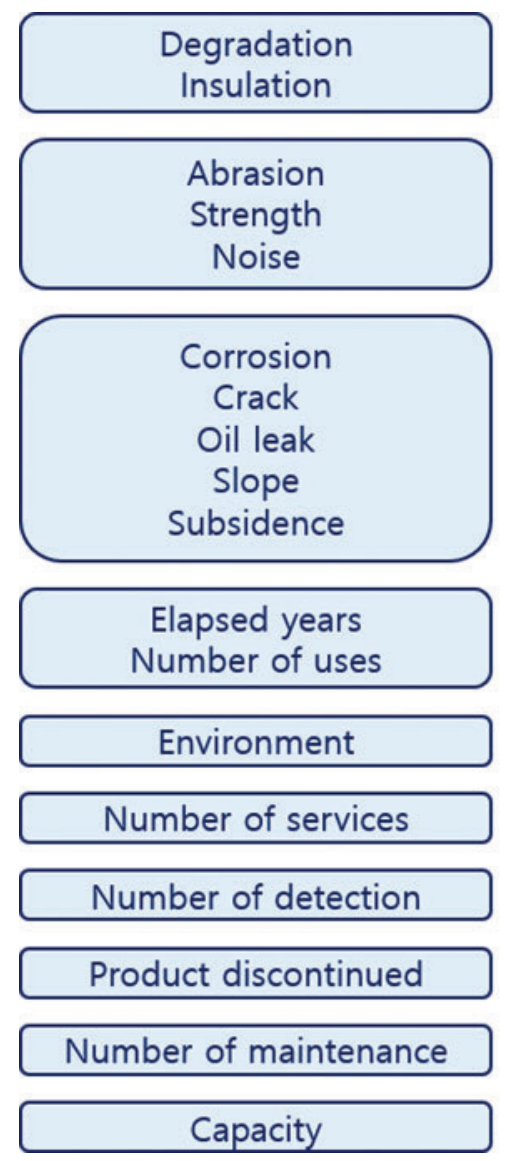

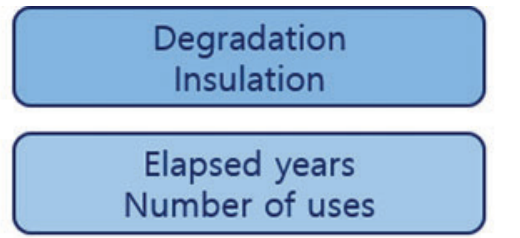

$14.63 \%$

$11.15 \%$

Product discontinued

$10.77 \%$

Number of detection

$10.68 \%$

Number of services

$10.03 \%$

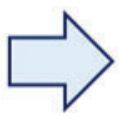

Number of maintenance

$9.83 \%$

Abrasion
Strength
Noise

$9.34 \%$

Environment

$9.25 \%$

Corrosion

Crack

Oil leak

Slope

Subsidence

$9.34 \%$

Capacity

Figure 7: Weight suggestion results 


\section{Conclusion}

This paper conducted a weight determination study on the maintenance detail criteria items with the aim of ensuring reliability and safety of railroad electrical facility systems in South Korea. safety, durability, and usability were selected as evaluation criteria, and the detailed criteria for maintenance of railroad electrical facility systems were divided into degradation, insulation, abrasion, strength, noise, corrosion, crack, oil leak, slope, subsidence, elapsed years, number of uses, environment, number of services, number of failure detection, product discontinued, number of maintenance, capacity. An alternative to the evaluation criteria was described as Design Structure Matrix, reflecting the opinions of railroad electrical system maintenance experts, and clustering of alternatives was performed through partitioning. As a result, it could be reduced to 10 alternatives with similar evaluation properties. To determine the weighting of the maintenance detail basis alternatives by the evaluation criteria, the weighting of the assessment criteria and the weighting of the basis alternatives were represented in fuzzy matrix, and the CC values were calculated by determining FPIRP and FNIRP. By normalizing the results for this, the weights of alternatives for each railroad electrical facility maintenance detail criteria item are presented as Percentage. As a result, degradation, insulation items were weighted the highest at $14.63 \%$, and capacity items were weighted the lowest at 5.34\%. A final comparison of this study's conclusions through peer review resulted in a valid opinion. The results of this study may contribute to the creation of a maintenance manual for electrical railway facilities systems to be carried out in the future. Also, the results of this may be contributed to the underlying research in carrying out maintenance activities to ensure the reliability and safety of railroad electrical facility systems. The limitation of this paper is how to select the scope of experts in electric railroad facility systems. Further research is also needed to determine the range of membership functions and survey scales for obtaining the Fuzzy matrix. Future research will change the process of drawing conclusions respectively. In addition, each conclusion will be compared and peer reviewed to carry out results verification and to produce reasonable procedures.

Funding Statement: This research was supported by a grant from R\&D Program of the Korea Railroad Research Institute, Republic of Korea.

Conflicts of Interest: The authors declare that they have no conflicts of interest to report regarding the present study.

\section{References}

[1] Ministry of Land, Infrastructure and Transport, "Basic plan for maintenance management of railway facilities," Korea: Ministry of land, Infrastructure and Transport, vol. 1, pp. 4-25, 2020.

[2] H. I. Kang, Y. S. Kim, J. S. Sim, H. G. Im, K. S. Ryu et al., "Maintenance optimization of the railway power facility," in Proc. the Korean Society for Railway, Korea, pp. 530-535, 2010.

[3] H. M. Kim and Y. H. Kim, "Analysis of railway infrastructure maintenance system," in Proc. the Korean Society for Railway, Korea, pp. 1523-1528, 2010.

[4] H. J. Park, Y. Park, H. S. Jung and H. C. Kim, "A comparative study on the railway electricity maintenance management," in Proc. the Korean Society for Railway, Korea, pp. 1202-1207, 2011.

[5] Y. S. Oh and B. K. Kim, "The study on the efficiency of conventional railway infrastructure maintenance on current status," in Proc. the Korean Society for Railway, Korea, pp. 2205-2216, 2011.

[6] S. G. Park, S. T. Song and C. M. Jeong, "Analysis of the status of the railway facility maintenance and its implications," in Proc. the Korean Society for Railway, Korea, pp. 383-389, 2013.

[7] K. M. Na, H. S. Jung, S. K. Shin and H. C. Kim, "Performance evaluation analysis program for electric railway facilities," Journal of the Korean Society for Railway, vol. 23, no. 6, pp. 542-550, 2020. 
[8] G. U. Kang, I. S. Jung, J. Y. Kim and M. B. Seo, "Preliminary study on the simulation for urban railway facility performance assessment," Journal of Korea Academy Industrial Cooperation Society, vol. 21, no. 3, pp. 190-198, 2020.

[9] T. H. Kim and J. C. Lee, "On an approach to developing the operational architecture for a railway safety information system," Journal of the Korea Safety Management \& Science, vol. 9, no. 6, pp. 9-17, 2007.

[10] K. H. Choi, Y. H. Kim, J. W. Lee, J. H. Song and K. Y. Song, "Technical review on the QRA of railway safety facilities," Journal of the Korea Safety Management \& Science, vol. 13, no. 3, pp. 1-8, 2011.

[11] Chen, C. T., "Extension of the TOPSIS for group decision-making under fuzzy environment," Fuzzy Set and Systems, vol. 114, issue no. 1, pp. 1-9, 2000.

[12] P. Kumar and R. S. Thakur, "An approach using fuzzy sets and boosting techniques to predict liver disease," computers," Materials \& Continua, vol. 68, no. 3, pp. 3513-3529, 2021.

[13] Y. B. Abushark, A. I. Khan, F. J. Alsolami, A. Almalawi, M. M. Alam et al., "Usability evaluation through fuzzy AHP-tOPSIS approach: Security requirement perspective," Computers, Materials \& Continua, vol. 68, no. 1, pp. 1203-1218, 2021.

[14] R. Mahapatra, S. Samanta, M. Pal, J. G. Lee, S. K. Khan et al., "Colouring of COVID-19 affected region based on fuzzy directed graphs," Computers, Materials \& Continua, vol. 68, no. 1, pp. 1219-1233, 2021.

[15] M. Jayalakshmi, L. Garg, K. Maharajan, K. Jayakumar, K. Srinivasan et al., "Fuzzy logic-based health monitoring system for COVID'19 patients," Computers, Materials \& Continua, vol. 67, no. 2, pp. 24312447, 2021.

[16] Y. Hafeez, S. Ali, N. Jhanjhi, M. Humayun, A. Nayyar et al., "Role of fuzzy approach towards fault detection for distributed components," Computers, Materials \& Continua, vol. 67, no. 2, pp. 1979-1996, 2021.

[17] Design Structure Matrix Conference, "Design structure matrix (DSM)," Germany, 2021. [Online]. Available: https://dsmweb.org/design-structure-matrix-dsm/. 